\title{
Angiomiolipoma nasal: reporte de dos casos y revisión de la literatura
}

\section{Nasal angiomyolipoma: report of two cases and literature review}

\author{
Nicolás Utrera Q. ${ }^{1}$, Fabiola Fuenzalida $O .^{2}$, Daniel Sotomayor J. ${ }^{3}$, \\ Felipe Gauna P. ${ }^{3}$, José Rojas B. ${ }^{3}$, Daniel Moyano M. ${ }^{3}$
}

${ }^{1}$ Facultad de Medicina de Universidad de Valparaíso. Valparaíso, Chile. ${ }^{2}$ Hospital Santo Tomás de Limache. Limache, Chile. ${ }^{3}$ Servicio de Otorrinolaringología del Hospital Carlos Van Buren. Universidad de Valparaíso. Valparaíso, Chile.

Los autores declaran no tener conflictos de interés.

Recibido el 26 de mayo de 2020. Aceptado el 7 de octubre de 2020

Correspondencia: Daniel Moyano M.

Almirante Señoret 151, Depto. 71

Valparaíso, Chile.

Email: daniel.moyano@ hotmail.com

\section{Resumen}

El angiomiolipoma es una lesión hamartomatosa, habitualmente de localización renal asociado a esclerosis tuberosa. La ubicación nasosinusal es extremadamente infrecuente y se puede presentar con obstrucción nasal y epistaxis. Se presentan dos casos de angiomiolipoma de cavidad nasal diagnosticados en el Hospital Carlos Van Buren de Valparaíso. Ambos casos se presentaron como masa nasal unilateral, se estudiaron con tomografía computada y fueron tratados con biopsia escisional, sin presentar recidivas durante su seguimiento. El anigiomiolipoma está compuesto por vasos sanguíneos, músculo liso y adipocitos. Existen diferencias entre el angiomiolipoma del riñón versus el de piel, cavidad oral y nasal (angiomiolipomas mucocutáneos); estos últimos son extremadamente infrecuentes, de menor tamaño, con presencia de agregados linfoides, negativos para antígeno específico de melanoma HMB-45 y sin asociación a esclerosis tuberosa. Existen pocos casos reportados en la literatura de esta patología, por lo que existe poca evidencia. Clínicamente, se presentan con obstrucción nasal, epistaxis recurrente, sensación de masa nasal. El estudio preoperatorio depende de su extensión y puede incluir una tomografía computada y/o angiografía para identificar su irrigación. El diagnóstico definitivo es anatomopatológico. Si se logra la escisión completa, no se han reportado recidivas, por lo que presenta un excelente pronóstico.

Palabras clave: Angiomiolipoma, nasosinusal, obstrucción nasal.

\begin{abstract}
Angiomyolipoma is a hamartomatous lesion, usually of a renal location associated with tuberous sclerosis. Nasosinusal location is extremely rare and can present with nasal obstruction and epistaxis. Two cases of angiomyolipoma of the nasal cavity diagnosed at the Carlos Van Buren Hospital in Valparaíso are presented. Both cases presented as a unilateral nasal mass, and were studied with computed tomography, and treated with an excisional biopsy, with no recurrences during follow-up. Anigiomyolipoma is composed of blood vessels, smooth muscle and adipocytes. There are differences between angiomyolipoma of the kidney versus skin, oral and nasal cavity (mucocutaneous angiomyolipomas). The latter are extremely infrequent, smaller in size, with the presence of lymphoid aggregates, negative for the HMB-45 specific melanoma antigen and without association with tuberous sclerosis. There are few cases reported in the literature of this pathology, so there is little evidence. Clinically, they present with nasal obstruction, recurrent epistaxis, sensation of nasal mass. The preoperative study depends on its extension and may include a computed tomography and/ or angiography to identify its irrigation. The definitive diagnosis is pathological. If complete excision is achieved, no recurrences have been reported, so it has an excellent prognosis.
\end{abstract}

Keywords: Angiomyolipoma, nasosinusal, nasal obstruction.

\section{Introducción}

Las neoplasias nasosinusales presentan muy baja incidencia, la suma de los tumores malignos y benignos a este nivel sumarían un total menor al 3\% de los tumores del tracto aerodigestivo superior ${ }^{1}$. Dada la inespecificidad del cuadro clínico de estos tumores se suele recurrir con frecuencia a pruebas de imagen e histopatológicas para el diagnóstico definitivo 
de estas lesiones ${ }^{2}$. El angiomiolipoma (AML) corresponde a una lesión hamartomatosa inusual cuya incidencia global bordea el $0,3 \%$ de la población ${ }^{3}$. Su composición consta de vasos sanguíneos de diferente tamaño, músculo liso y adipocitos, tal como lo indica su nombre ${ }^{4}$. $\mathrm{Su}$ aparición en un $90 \%$ de los casos es esporádica, pero en un $10 \%$ podría presentarse de manera hereditaria, apareciendo estos últimos asociados a pacientes con esclerosis tuberosa, desorden autosómico-dominante con predisposición a formar tumores hamartomatosos en distintas localizaciones ${ }^{5}$. Dentro de dichas localizaciones, las más frecuentes son la renal y hepática, siendo otras localizaciones, tales como la nasal, extremadamente infrecuentes. De estos últimos, existen menos de 20 casos reportados en la literatura ${ }^{6}$. La presentación clínica es similar a otras masas nasales unilaterales, por lo que el estudio histológico e inmunohistoquímico es fundamental para el diagnóstico ${ }^{3}$. El manejo habitual es la biopsia escisional, no existiendo reportes de recidiva en la literatura ${ }^{7}$.

Presentamos 2 casos de angiomiolipoma nasal diagnosticados en el Hospital Carlos Van Buren de Valparaíso, Chile. En ambos casos se obtuvo consentimiento informado para reporte de casos clínicos aprobado por el comité ético científico del Servicio de Salud Valparaíso-San Antonio.

\section{Caso Clínico 1}

Paciente de sexo femenino de 70 años, con antecedentes de hipotiroidismo y dislipidemia. Consultó por cuadro de 10 años de evolución caracterizado por obstrucción nasal izquierda permanente que aumentó en los últimos dos años, sin otros síntomas nasosinusales. Al examen físico se observó una lesión exofítica en vestíbulo nasal izquierdo de $1,0 \mathrm{~cm}$ x $1,5 \mathrm{~cm}$ de diámetro, de base pediculada, color violáceo y aspecto vascularizado, de superficie lisa y blanda a la tracción, que nacía de la mucosa del ala nasal. El resto del examen físico no presentaba alteraciones. Se realizó nasofibroscopía, objetivándose la lesión anterior al cornete inferior, sin extensión al resto de la cavidad nasal. Se realizó biopsia escisional, apreciándose lesión violácea vascularizada con amplia base de im- plantación a nivel de cartílago alar. El estudio histológico e inmunohistoquímico diferido informó un angiomiolipoma nasal, con tinción HMB45 negativa. Durante 4 meses de seguimiento sin evidencias de recidiva tumoral.

\section{Caso Clínico 2}

Paciente de sexo masculino de 84 años, asmático. Consultó por cuadro de un año de evolución de obstrucción nasal progresiva en fosa nasal izquierda, asociado a descarga posterior. Al examen físico se observó un tumor exofítico rojo violáceo que ocupa todo el vestíbulo nasal izquierdo. A la nasofibroscopía se observó este tumor pediculado, algo friable, que nace de cornete inferior y que contacta septo $\sin$ infiltrarlo (Figura 1).

Se solicitó una tomografía computada de cavidades paranasales con contraste donde se observa una imagen nodular que oblitera región vestibular, de $24 \mathrm{~mm} \times 12 \mathrm{~mm}$, con vascularización heterogénea dependiente del cornete inferior, sin invasión al resto de la cavidad nasal. Sin signos de remodelación ósea. Se realizó biopsia escisional con técnica fría, resecando completamente el tumor desde su base de implantación. La biopsia rápida informó un pólipo nasal angiomatoso, sin lesiones en base de implantación. La biopsia diferida informó un angiomiolipoma nasal (Figura 2). $\mathrm{Al}$ año y dos meses de seguimiento se encuentra sin sintomatología ni evidencias de recidiva.

\section{Discusión}

El angiomiolipoma corresponde a una lesión neoplásica benigna, que en la mayoría de los casos se origina a nivel renal, pudiendo estar en menor frecuencia en otras localizaciones, tales como hígado, mediastino, corazón, pared vaginal, cordón espermático, trompas de Falopio, cavidad nasal, faringe y piel $^{8}$. Dentro de las localizaciones inusuales del angiomiolipoma, la más documentada es la ubicación en cavidad nasal ${ }^{4}$. En estos casos, los AML nasales se presentan con mayor frecuencia en el sexo masculino con una relación de hasta 3:1 con rangos de edad que van entre los 34-88 años?. 


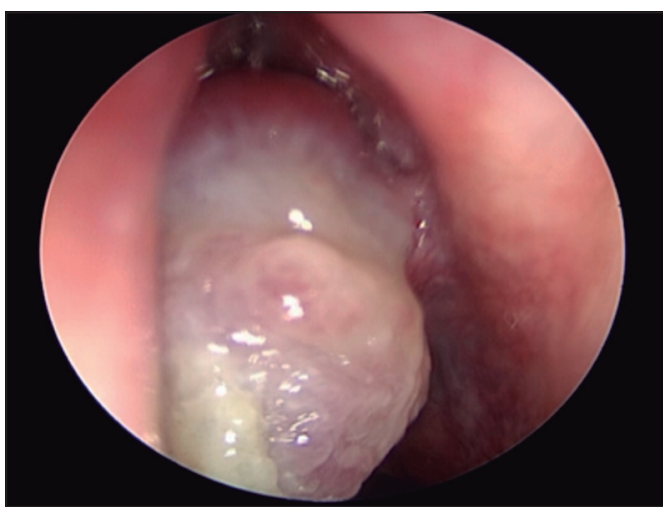

Figura 1. Nasofibroscopia de cavidad nasal izquierda que evidencia lesión exofitica violácea que nace de la mucosa (caso clínico $n^{\circ} 2$ ).

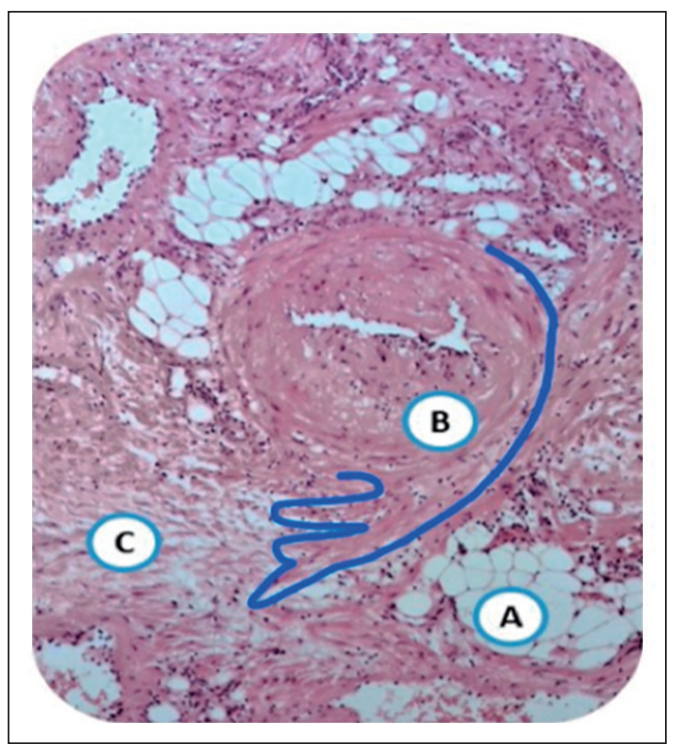

Figura 2. Corte histológico de lesión nasal biopsiada. A: Tejido adiposo. B: Vasos sanguíneos arteriolares con fenómeno de extensión de musculatura lisa. C: Músculo liso.

El primer caso reportado data de 1988, con un paciente de 52 años en Arabia Saudita que consultó por un cuadro de un año de evolución de obstrucción nasal derecha y epistaxis recurrente $^{10}$. Desde entonces, sólo se han descrito menos de veinte casos más hasta la fecha; en nuestro país solo existe un caso reportado. Cuando se analiza la localización nasal, los AML se han descrito en diversas ubicaciones, como pared lateral, techo nasal, vestíbulo nasal, pliegue nasolabial, cavidad nasal, coanal y a nivel del meato inferior ${ }^{7}$.
La presentación clínica más frecuente de los AML nasales es obstrucción nasal y epistaxis recurrente, siendo otros síntomas también encontrados como sensación de masa nasal, cuerpo extraño rinofaríngeo y roncopatía $y$, finalmente, pudiese encontrarse también como un hallazgo imagenológico ${ }^{11}$. El diagnóstico definitivo de esta lesión es anatomopatológico $^{3}$. Los angiomiolipomas de la piel y de la cavidad nasal se componen comúnmente de vasos sanguíneos de varios tamaños, células musculares lisas, células grasas y tienen varias diferencias con los angiomiolipomas renales y hepáticos ${ }^{4}$. Una de las diferencias anatomopatológicas más importantes es que están formados sólo por células musculares lisas maduras y el marcador HMB45 es negativo. Además, en estos tumores existen agregados linfocíticos que son raros de encontrar en los AML renales y hepáticos ${ }^{4}$. Estas características que los diferencian de los hepáticos y renales son idénticas a las de los encontrados en la cavidad oral y en la faringe, lo que ha llevado a denominarlos angiomiolipomas mucocutáneos, término acuñado por Watanabe y Suzuki. Dentro de las características distintivas de los angiomiolipomas mucocutáneos, destaca la no asociación con esclerosis tuberosa y la presentación casi exclusiva en adultos mayores. También destaca el tamaño, donde los AML mucocutáneos presentan dimensiones que en promedio son menores a $40 \mathrm{~mm}$, versus los renales y hepáticos que por lo general son de mayor tamaño, superando los 40-45 $\mathrm{mm}^{4}$.

Otra forma en la que han sido clasificados estos tumores es en la categoría de tumores de células epiteloides perivasculares (PEComas en literatura inglesa), que incluye diversas lesiones cuyo sello es ser tumores mesenquimáticos con presencia de las células epiteloides perivasculares, agrupando los angiomiolipomas, linfangioleiomiomatosis, tumor de células claras del pulmón, tumor miomelanocítico de células claras, entre otros ${ }^{6}$. Los PEComas presentan citoplasmas granulares eosinófilos claros a pálidos y un núcleo central redondeado/ovalado con nucléolo discreto y la presencia de marcadores inmunohistoquímicos melanocíticos y mioides ${ }^{12}$.

Pese a lo anterior, a nivel histológico, existen ciertas características propias de los AML mucocutáneos que los diferencian dentro del 
grupo de los PEComas, tales como la ausencia de células epiteloides de músculo liso, con la presencia exclusiva de células musculares maduras, además de la presencia de agregados linfoides. A nivel de tinción, demuestran ser negativos para el antígeno específico de melanoma HMB-45, positivos para SMA actina de músculo liso y positivos para las proteínas S100 a nivel de adipocitos $3,7,9,12,13$.

En cuanto al estudio complementario de estas lesiones, la imagenología puede ayudar a evaluar la ubicación de la lesión y determinar el compromiso óseo, siendo la tomografía computada el estudio más comúnmente utilizado $^{6}$. En ocasiones se puede requerir el estudio preoperatorio con angiografía y eventual embolización para disminuir el riesgo de sangrado, aunque no existen reportes de sangrado excesivo en estos pacientes ${ }^{4,6}$. Como diagnóstico diferencial a nivel clínico, debiese considerarse toda masa nasal unilateral, tales como pólipos, papilomas y angiofibromas nasales, entre otros. A nivel anatomopatológico, el principal diagnóstico diferencial es el angiomioma ${ }^{2}$. Respecto al tratamiento y seguimiento, no se han reportado recidivas ni metástasis tras la escisión tumoral completa, por lo que este sigue siendo el manejo de elección para este tipo de tumor.

\section{Conclusión}

El angiomiolipoma nasal es una lesión tumoral hamartomatosa altamente infrecuente, existiendo menos de 20 casos reportados en la literatura. Su presentación clínica es similar a otras masas nasosinusales, por lo que el estudio imagenológico es importante para su diagnóstico diferencial y evaluar su extensión. El diagnóstico definitivo es histológico e inmunohistoquímico. El tratamiento de elección es la biopsia escisional, no existiendo casos de recidiva reportados en la literatura. A diferencia del angiomiolipoma renal y hepático, la ubicación nasal no presenta relación con esclerosis tuberosa. Un alto índice de sospecha es necesario para diagnosticar este tipo de tumores.

\section{Bibliografía}

1. Quitral R, Rahal M, Morales I, Daszenies C, Vallejos MP. Tumores malignos de nariz y cavidades paranasales. Revisión de 11 años. Rev Otorrinolaringol Cir Cabeza Cuello. 2003;63(1):21-28.

2. Ibáñez JA, Segura J, Gómez MJ, García-Eloy C. Angiomiolipoma Nasal. Acta Otorrinolaringol Esp. 2014;65(3):205-207.

3. Iwata A, Friedmann D, Kaplan J, Wang B, Lebowitz R. A Man With Recurrent Right-Sided Epistaxis. JAMA Otolaryngol Head Neck Surg. 2013;139(10):1067-1068.

4. Watanabe K, Suzuki T. A Report of 2 Cases Arising in the Nasal Cavity. Arch Pathol Lab Med. 1999;123(9):789-792.

5. Bhatt J, Richard P, Kim N, et al. Natural History of Renal Angiomyolipoma (AML): Most Patients with Large AMLs $>4 \mathrm{~cm}$ Can Be Offered Active Surveillance as an Initial Management Strategy. Eur Urol. 2016;70(1):85-90.

6. Ameen SA, Salina H, Zahedi FD, PrimuharsaPutra SH, Masir N. Nasal Angiomyolipoma (AML) Mimicking Juvenile Nasopharyngeal Angiofibroma. Iran J Otorhinolaryngol. 2019;31(104):191-195.

7. Cancino M, Castillo J, Cantero D. Angiomiolipoma de cavidad nasal. Reporte de un caso y revisión de la literatura. Rev Otorrinolaringol Cir Cabeza Cuello. 2017;77:157-163.

8. Ahn SH, Lee YJ, Kim CH, Chang JH. Angiomyolipoma of the Nasal Cavity Resected with Preoperative Angio-Embolization. Korean J Otorhinolaryngol-Head Neck Surg. 2013;56(8):528531.

9. Aleem MA, Fatima A, Kumudachlam P, Priyadarshini R. Nasal angiomyolipoma: Report of two cases of an extremely rare entity. Indian J Pathol Microbiol. 2017;60(3):393-395.

10. Dawlatly EE, Anim JT, el-Hassan AY. Angiomyolipoma of the nasal cavity. J Laryngol Otol. 1988;102(12):1156-1158.

11. Erkiliç S, Koçer NE, Mumbuç S, Kanlikama M. Nasal angiomyolipoma. Acta Otolaryngol. 2005; 125(4):446448.

12. Bandhlish A, Leon Barnes E, Rabban JT, McHugh JB. Perivascular Epithelioid Cell Tumors (PEComas) of the Head and Neck: Report of Three Cases and Review of the Literature. Head Neck Pathol. 2011;5(3):233-240.

13. Go JH. Angiomyolipoma of the Nasal Cavity. Korean J Pathol. 2005;39:284-286. 